\title{
Characterization of cocaine addiction-like behavior in heterogeneous stock rats
}

Giordano de Guglielmo ${ }^{1 \# *}$, Lieselot LG Carrette ${ }^{1 \#}$, Marsida Kallupi ${ }^{1}$, Molly Brennan ${ }^{1}$, Brent

Boomhower $^{1}$, Lisa Maturin ${ }^{1}$, Dana Conlisk ${ }^{2}$, Sharona Sedighim ${ }^{1}$, Lani Tieu ${ }^{1}$, McKenzie J

Fannon $^{1}$, Angelica Martinez ${ }^{1}$, Nathan Velarde ${ }^{1}$, Jenni Kononoff ${ }^{2}$, Adam Kimbrough ${ }^{1}$, Sierra

Simpson ${ }^{1}$, Lauren C Smith ${ }^{1,2}$, Kokila Shankar ${ }^{1,2}$, Caitlin Crook ${ }^{1}$, Alicia Avelar ${ }^{1}$, Paul

Schweitzer ${ }^{1}$, Leah C. Solberg Woods ${ }^{3}$, Abraham A. Palmer ${ }^{1,4}$ and Olivier George ${ }^{1 *}$

${ }^{1}$ Department of Psychiatry, University of California, San Diego, La Jolla CA 92093, USA.

${ }^{2}$ Department of Neuroscience, The Scripps Research Institute, La Jolla, CA 92037, USA.

${ }^{3}$ Department of Internal Medicine, Section on Molecular Medicine, Wake Forest University School of Medicine, Winston-Salem, NC 27157, USA.

${ }^{3}$ Institute for Genomic Medicine, University of California, San Diego, La Jolla CA 92093, USA.

\# These 2 authors contributed equally

*Corresponding authors

Dr. Giordano de Guglielmo and Dr. Olivier George, Department of Psychiatry, University of California San Diego, School of Medicine, La Jolla, CA 92093, USA. Tel: +18582465538. Email: gdeguglielmo@health.ucsd.edu olgeorge@health.ucsd.edu

Funding and disclosure

The authors declare no conflict of interest. 


\begin{abstract}
Twin studies suggest that approximately $50 \%$ of the vulnerability to cocaine use disorder is determined by genetic factors, but genome-wide association studies (GWAS) in humans have only begun to identify specific genes that confer this risk. The identification of a set of single nucleotide polymorphisms (SNPs) associated with increased vulnerability to develop compulsive cocaine use represents a major goal for understanding of the genetic risk factors to cocaine use disorder and facilitating the identification of novel druggable targets.

Here we characterized addiction-like behaviors in heterogeneous stock (HS) rats, a unique outbred strain of rats characterized by high genetic variability that has been developed to mimic genetic variability in humans. HS rats were allowed to self-administer cocaine 6h/daily for 14 days. Animals were also screened for compulsive cocaine use, using progressive-ratio (PR) and responding despite adverse consequences (contingent foot shocks). To minimize cohort-specific effects, we used large cohorts $(n=46-60)$ and normalized the level of responding within cohorts using a $Z$-score. To take advantage of the three behaviors related to compulsive intake and further identify subjects that are consistently vulnerable $v s$. resilient to compulsive cocaine use we computed an Addiction index by averaging normalized responding (Z-scores) for the three behavioral tests. Results showed high individual variability between vulnerable and resilient rats that is likely to facilitate detection of gene variants associated with vulnerable vs. resilient individuals. Such data will have considerable translational value for designing pharmacogenetic studies in humans.
\end{abstract}




\section{Introduction}

Cocaine use disorder (CUD) remains a serious public health problem in the USA, with 2.2 million regular cocaine users in 2019 and over a million individuals with CUD in the past year (Samsha). The use of cocaine is associated with substantial morbidity and elevated rates of health care utilization (Butler, Rehm, \& Fischer, 2017), and over the last 10 years, the number of cocaine-related deaths has quadrupled (Hedegaard, Bastian, Trinidad, Spencer, \& Warner, 2018). At the moment, there are no FDA-approved pharmacological treatments for cocaine use disorder (Kampman, 2019). A key and unanswered question for addiction research remains why casual patterns of drug consumption escalate to problematic patterns associated with high motivation and compulsivity typical for a substance use disorder in some individuals, but not others. A better understanding and characterization of the individual differences in the propensity to develop addiction-like behaviors (Vowles et al., 2015) could help into the development of new therapies and in the identification of new pharmacological targets. Vulnerability to substance abuse and addiction has a heritable component. In particular, family and twin studies demonstrate that about $50 \%$ of the vulnerability to develop cocaine use disorder is determined by genetic factors (Agrawal et al., 2012; Ducci \& Goldman, 2012; Kreek, Bart, Lilly, LaForge, \& Nielsen, 2005). This genetic contribution is polygenic, with many factors contributing vulnerability or resilience interacting together. In the past decade, advances in genetics have provided critical clues in the search for the molecular basis of alcohol use and abuse. Human genome-wide association studies (GWAS) have expanded dramatically in size and sophistication, which has led to hundreds of loci being implicated in a range of addiction-related traits (Erzurumluoglu et al., 2020; Sanchez-Roige, Fontanillas, et al., 2019; Sanchez-Roige, Palmer, et al., 2019; Walters et al., 2018; H. Zhou et al., 2020); however, the vast majority of genes that confer risk remain unknown, since clinical studies on cocaine use disorder face several difficulties, including imprecision of self-reported drug addiction measures, lack of longitudinal analyses of behavior, lack of biological samples, lack of control over environmental variables, and qualitative vs. quantitative measures. Therefore, the genes/alleles that mediate individual differences in the effect of cocaine and opioids and the biological mechanisms responsible for developing addiction-like behaviors are still poorly defined (Hart, de Wit, \& Palmer, 2013; Klepstad et al., 2011). In parallel with the advances in human genetics, the methodology for GWAS in mice and rats has also been revolutionized over the past decade. Prior 
efforts were hindered by populations that did not have sufficient recombination, inadequate genotyping platforms, and limitations in statistical methodology. One key development has been the adoption of populations in which inbred founders have been allowed to undergo numerous generations of recombination, which breaks founder haplotypes into small intervals, supporting the fine mapping of associated loci. Developments in genotyping technology and statistical approaches have also set the stage for GWAS in mice and rats that identify small genetic intervals, often containing one or a few genes.

Preclinical settings allow for longitudinal analyses with qualitative and quantitative measures that can be highly standardized and replicated while maintaining a controlled environment. Moreover, genetic manipulation and thorough dissections are possible. A major achievement in the field of cocaine addiction has been the development of the model of extended access to cocaine self-administration that has shown high face, predictive/postdictive, and construct validity and is thus highly relevant to cocaine use disorders (Edwards \& Koob, 2013; O. George, G. F. Koob, \& L. F. Vendruscolo, 2014; O. George, C. D. Mandyam, S. Wee, \& G. F. Koob, 2008). It is associated with neuroadaptations that are also observed in humans with cocaine use disorder (Briand, Gross, \& Robinson, 2008; O. George et al., 2008).

Here we provide a comprehensive characterization of cocaine addiction-like behaviors in $>500$ heterogeneous stock (HS) rats. HS rats are the most highly recombinant rat intercross available and are a powerful tool for genetic studies (Chitre et al., 2020; Solberg Woods \& Palmer, 2019). HS rats were created by interbreeding eight inbred strains and maintaining them as an outbred population in a way that minimizes inbreeding, thereby maximizing genetic diversity within the colony (Solberg Woods \& Palmer, 2019). All eight inbred founders of the HS have been deeply sequenced by several labs. With each generation of outbreeding, new recombinations accumulate, allowing for genetic mapping to increasingly smaller intervals. Thus, each HS individual represents a mosaic of haplotypes from the eight inbred founder strains. Critically, each HS rat is genetically and phenotypically unique. The rats were exposed to extended access of cocaine self-administration combined with behavioral characterization of compulsivity using progressive ratio responding and responding despite adverse consequences (contingent foot shocks). A comprehensive characterization of irritability like behavior during acute withdrawal was also performed. The results of the different behavioral tests were normalized and computed 
into an Addiction Index that allowed to identify animals vulnerable or resilient to compulsive cocaine use.

\section{METHODS}

Detailed procedures can be found in the George lab protocol repository on protocols.io (https://www.protocols.io/workspaces/george-lab).

Animals. HS rats (Rat Genome Database NMcwiWFsm \#13673907, sometimes referred to as $\mathrm{N} / \mathrm{NIH}$ ) created to encompass as much genetic diversity as possible at the NIH in the 1980's by outbreeding eight inbred rat strains (ACI/N, BN/SsN, BUF/N, F344/N, M520/N, MR/N, WKY/N and WN/N) were provided by Dr. Leah Solberg Woods (Medical College of Wisconsin, now at Wake Forest University School of Medicine; $n=600$ ). To minimize inbreeding and control genetic drift, the HS rat colony consists of more than 64 breeder pairs and is maintained using a randomized breeding strategy, with each breeder pair contributing one male and one female to subsequent generations. To keep track of the rats, their breeding, behavior, organs and genomic info, each rat received a chip with an RFID code. Rats were shipped at 3-4 weeks of age, kept in quarantine for 2 weeks and then housed two per cage on a $12 \mathrm{~h} / 12 \mathrm{~h}$ reversed light/dark cycle in a temperature $\left(20-22^{\circ} \mathrm{C}\right)$ and humidity $(45-55 \%)$ controlled vivarium with ad libitum access to tap water and food pellets (PJ Noyes Company, Lancaster, NH, USA). All of the procedures were conducted in strict adherence to the National Institutes of Health Guide for the Care and Use of Laboratory Animals and were approved by the Institutional Animal Care and Use Committees of The Scripps Research Institute and UC San Diego.

Drugs. Cocaine $\mathrm{HCl}$ (National Institute on Drug Abuse, Bethesda, MD) was dissolved in 0.9\% sterile saline and administered intravenously at a dose of $0.5 \mathrm{mg} / \mathrm{kg} / \mathrm{infusion}$.

Intravenous Catheterization. Rats were anesthetized with vaporized Isoflurane (1-5\%). Intravenous catheters were aseptically inserted into the right jugular vein using the procedure described previously (Kallupi et al., 2020). Catheters consisted of Micro-Renathane tubing (18 cm, 0.023-inch inner diameter, 0.037-inch outer diameter; Braintree Scientific, Braintree, MA, 
USA) attached to a 90 degree angle bend guide cannula (Plastics One, Roanoke, VA, USA), embedded in dental acrylic, and anchored with mesh (1 mm thick, $2 \mathrm{~cm}$ diameter). Tubing was inserted into the vein following a needle puncture $(22 \mathrm{G})$ and secured with a suture. The guide cannula was punctured through a small incision on the back. The outside part of the cannula was closed off with a plastic seal and metal cover cap, which allowed for sterility and protection of the catheter base. Flunixin $(2.5 \mathrm{mg} / \mathrm{kg}$, s.c.) was administered as analgesic, and Cefazolin (330 $\mathrm{mg} / \mathrm{kg}$, i.m.) as antibiotic. Rats were allowed three days for recovery prior to any selfadministration. They were monitored and flushed daily with heparinized saline $(10 \mathrm{U} / \mathrm{ml}$ of heparin sodium; American Pharmaceutical Partners, Schaumberg, IL, USA) in 0.9\% bacteriostatic sodium chloride (Hospira, Lake Forest, IL, USA) that contained $52.4 \mathrm{mg} / 0.2 \mathrm{ml}$ of Cefazolin.

\section{Behavioral Testing}

Operant self-administration. Self-administration (SA) was performed in operant conditioning chambers $(29 \mathrm{~cm} \times 24 \mathrm{~cm} \times 19.5 \mathrm{~cm}$; Med Associates, St. Albans, VT, USA $)$ that were enclosed in lit, sound-attenuating, ventilated environmental cubicles. The front door and back wall of the chambers were constructed of transparent plastic, and the other walls were opaque metal. Each chamber was equipped with two retractable levers that were located on the front panel. Each session was initiated by the extension of two retractable levers into the chamber. Drugs (cocaine, $0.5 \mathrm{mg} / \mathrm{kg}$ per infusion in saline) were delivered through plastic catheter tubing that was connected to an infusion pump. The infusion pump was activated by responses on the right (active) lever that were reinforced on fixed ratio (FR) 1 schedule, with the delivery of $0.1 \mathrm{ml}$ of the drug per lever press over $6 \mathrm{~s}$ followed by a 20-s timeout period that was signaled by the illumination of a cue light above the active lever and during which active lever presses did not result in additional infusions. Responses on the left inactive lever were recorded but had no scheduled consequences. Fluid delivery and behavioral data recording was controlled by a computer with the MED-PC IV software installed. Initially, rats were trained to self-administer cocaine in 10 short access (ShA) sessions (2h/day). At the end of the ShA phase the animals were exposed to 14 extended access sessions (LgA, 6 h/day, 5 days/week) in order to measure escalation of drug intake. 
Progressive Ratio Testing. Rats were tested on a progressive ratio (PR) schedule of reinforcement at the end of each phase (twice after $\operatorname{LgA}$, see Fig 1 for detailed timeline), in which the response requirements for receiving a single reinforcement increased according to the following 1, 2, 4, 6, 9, 12, 15, 20, 25, 32, 40, 50, 62, 77, 95, 118, 145, 178, ... The breakpoint was defined as the last ratio attained by the rat prior to a 60 -min period during which a ratio was not completed, which ended the experiment.

Compulsive-like responding using contingent foot shock. A 1-hour footshock punishment testing was conducted between progressive ratio tests following the same parameters as the cocaine self-administration session. Punishment testing followed the same FR1 20-second timeout reinforcement schedule with contingent footshock $(0.3 \mathrm{~mA}, 0.5 \mathrm{sec})$ paired with $30 \%$ of the cocaine infusions.

Irritability-like behavior (Bottle Brush Test). The bottle brus test consisted of ten $10 \mathrm{sec}$ trials with $10 \mathrm{sec}$ intertrial intervals in plastic cages $(27 \mathrm{~cm} \times 48 \mathrm{~cm} \times 20 \mathrm{~cm})$ with clean bedding. The rats were placed in the back of the cage and a bottle-brush was rotated rapidly toward the rat's whiskers. Both aggressive responses (smelling, biting, boxing, following, and exploring the bottle-brush) and defensive responses (escaping, digging, jumping, climbing, defecation, vocalization, and grooming) were recorded by three trained observers in real-time. Total aggressive and defensive scores were calculated for each animal based on the average score of the observers. Both aggressive and defensive behaviors were summed to calculate the total irritability score. Irritability-like behavior reflects a composite measure of aggressive vs. defensive responses. (Kimbrough et al., 2017a). The test was performed after surgery (baseline) and in withdrawal $18 \mathrm{~h}$ after the last $\mathrm{LgA}$ session.

Statistical Analyses. Data were analyzed using Prism 9.0 software (GraphPad, San Diego, CA, USA). Self-administration data were analyzed using repeated-measures analysis of variance (ANOVA) followed by Bonferroni post-hoc tests when appropriate. For pairwise comparisons, data were analyzed using the Student's $t$-test. Correlations were calculated using Pearson $r$ analysis. The data are expressed as mean \pm SEM unless otherwise specified. Values of $\mathrm{p}<0.05$ were considered statistically significant. 


\section{Results}

\section{Evaluation of Individual Differences in Addiction-Like Behaviors.}

We assessed addiction-like behaviors in HS rats self-administering cocaine according to the standardized protocol shown in Figure 1A. Animals were tested in 12 cohorts with N=46-60 each. Thirty-four animals were excluded from the analysis because they lost their catheter patency before the end of the behavioral protocol. After catheterization surgery and recovery, the rats were trained to self-administer cocaine in 10 daily $2 \mathrm{~h}$ short access (ShA) sessions and then in 14 daily 6h long access (LgA) sessions. Over the course of the extended access of cocaine selfadministration phase, the animals showed escalation of intake, as measured by the significant increase of cocaine rewards from day 3 of LgA onwards vs the first day of LgA (Fig. 1B, p < 0.001 after one way ANOVA, followed by Bonferroni post hoc comparisons). The number of cocaine infusions in the last three days of LgA was averaged for each rat in order to compare the levels of cocaine intake at the end of the behavioral protocol in the whole population (Fig. 1C). The results showed a bimodal distribution with $20 \%$ of rats maintaining very low levels of intake, below 50 infusions per session (that corresponded to $\sim 8$ infusions/h, levels normally achieved during ShA, see Fig 1B) and $80 \%$ of the rats escalating their cocaine intake over the course of the extended access protocol. Motivation for cocaine was tested both after ShA and LgA, using a progressive ratio (PR) test (Fig. 1C). The number of cocaine infusions significantly increased after extended access of cocaine self-administration, compared to the levels at the end of the short access phase (t-test, $\mathrm{p}<0.001)$. Of note, also here a bimodal distribution can be observed, particularly after LgA. Compulsive-like behavior was tested by pairing the cocaine reward with foot shock (30\% contingency). Figure 1D shows the wide variability of HS rats on compulsive cocaine intake. Finally, cocaine-induced withdrawal was assessed by measuring irritability-like behavior using the bottle brush test (Kimbrough et al., 2017b). Irritability like behavior was measured at the beginning and at the end of the behavioral paradigm (pre- and post-cocaine, Fig. 1E) and the differences from baseline were compared to data obtained from age-matched naïve animals. Irritability like behavior was increased in cocaine rats during withdrawal compared to naïve animals as demonstrated by the increased total irritability score $(p<0.001$ vs naïve after t-test). When breaking the total irritability score in defensive and 
aggressive responses we found that animals with a history of cocaine increased their aggressive behaviors $(\mathrm{p}<0.001)$ but not their defensive behaviors, compared to their naïve counterparts.

A)

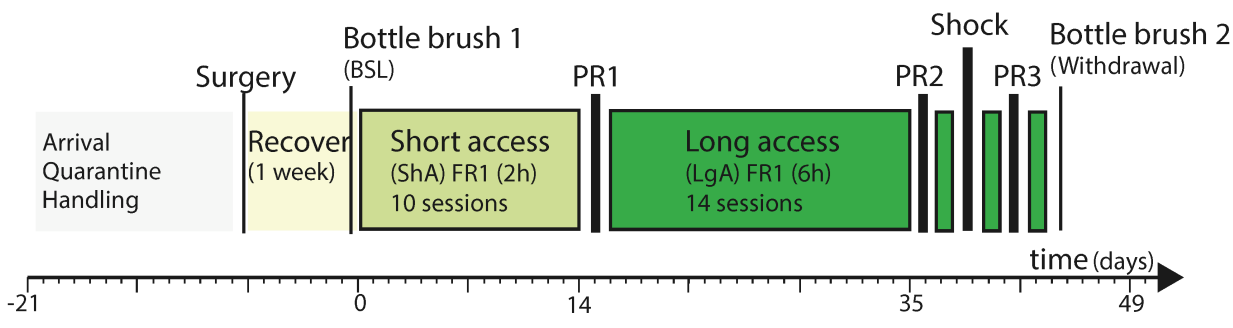

B)
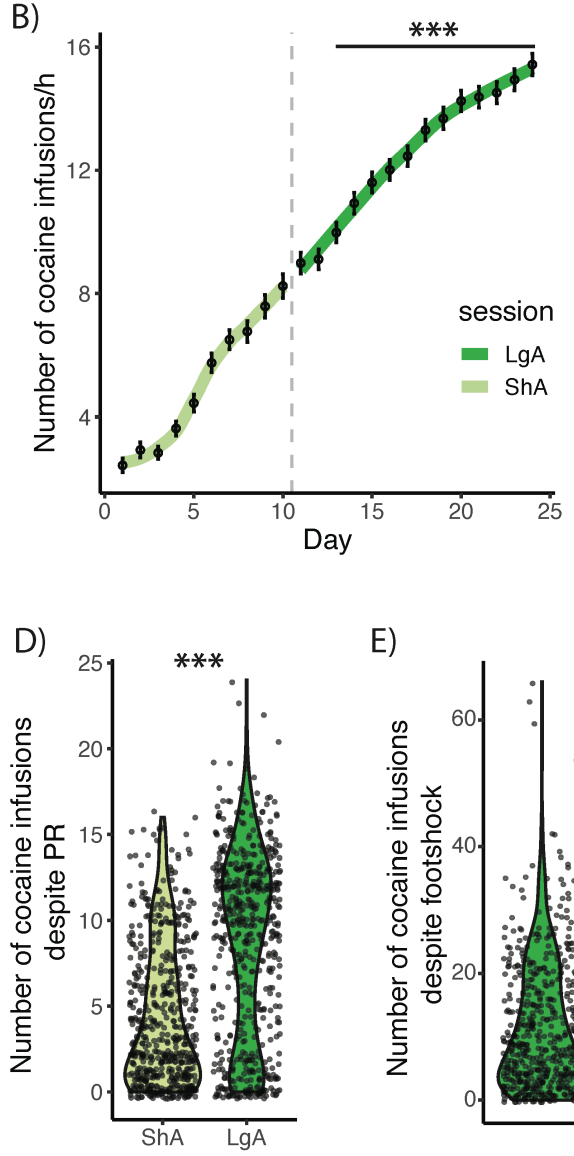

E)

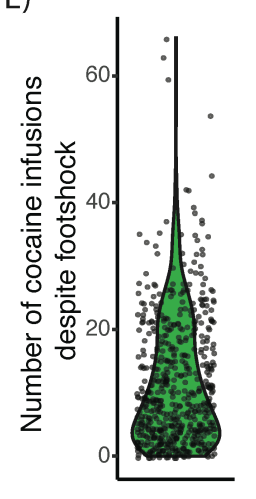

C)

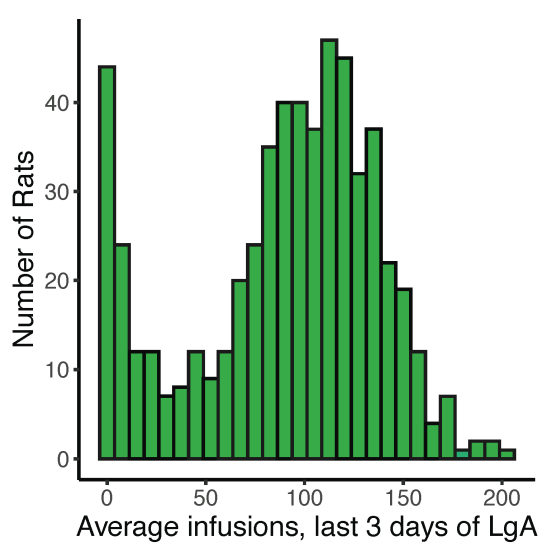

F)

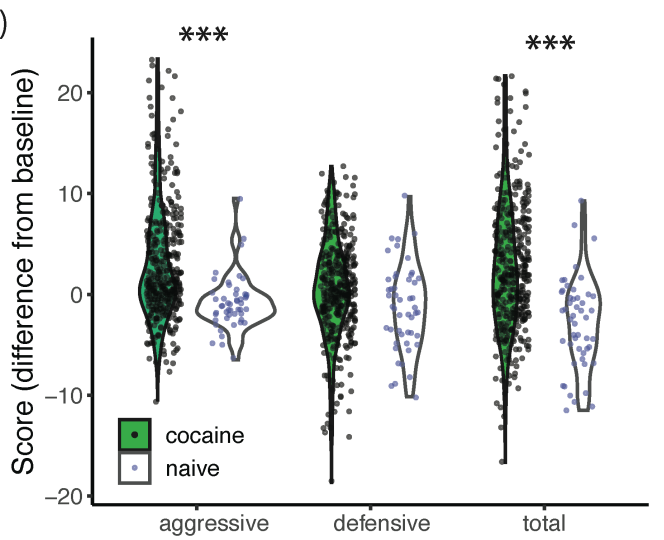

Figure 1. individual differences in addiction-behaviors in HS rats following intravenous cocaine selfadministration. A) Timeline of the behavioral paradigms. B) Number of cocaine infusions in the first hour of cocaine self-administration during short ( $2 h, S h A)$ and long ( $6 h, L g A)$ access ( $N=567,{ }^{* * *} p<0.001$ vs the first $L g A$ session). C) Average number of daily infusions for the last 3 days of $\operatorname{Lg}(N=567)$. D) Violin plot of number of cocaine infusions under progressive ratio (PR) test after ShA and $\operatorname{LgA}\left(N=564,{ }^{* * *} p<0.001\right)$. E) Number of infusions despite footshock after $\operatorname{LgA}(N=567)$. F) Difference in irritability scores after $\operatorname{Lg} A$ and at baseline $\left(N=380+49\right.$ naive, ${ }^{* * *} p<$ 0.001 vs naive). 


\section{Sex differences amongst addiction-like behaviors}

Major sex differences were observed with females acquiring cocaine self-administration faster and at higher levels than males under short access condition (2 h/day, Fig. 2A) and during the extended access (6 h/day, Fig. 2A). The two-way ANOVA with sex as between factor and sessions as within factor showed a significant effect of sex $(p<0.001)$, sessions $(p<0.001)$ and of the interaction sex*sessions $(\mathrm{p}<0.001)$. The Bonferroni post hoc test demonstrated that females self-administered more cocaine compared to males throughout the whole behavioral protocol ( $p$ $<0.01$ ). Male rats showed a slightly faster escalation of intake (significant differences from day 1 of $\operatorname{LgA}$ started on day 3) compared to females (significant differences from day 1 of $\operatorname{LgA}$ started on day 4 Fig. 2A). In the progressive ratio test, the two-way ANOVA with sex as between factor and time as within factor showed a significant effect of $\operatorname{sex}(p<0.05)$, time $(p<0.001)$ but not the interaction sex*time ( $\mathrm{p}=\mathrm{NS}$ ), confirming that motivation for cocaine was increased in both sexes over time from ShA to LgA (time, $\mathrm{p}<0.01$ ) and that females had significantly higher motivation for cocaine compared to males ( $\mathrm{sex}, \mathrm{p}<0.05$ ) independently from the lenght of access to cocaine (Fig. 2C) . Similar results were found for compulsive-like behavior, with females obtaining more infusions despite adverse consequences ( $p<0.001$ after t-test, Fig. 2D). We did not detect any sex-differences in irritability-like behavior (Fig. 2E). 

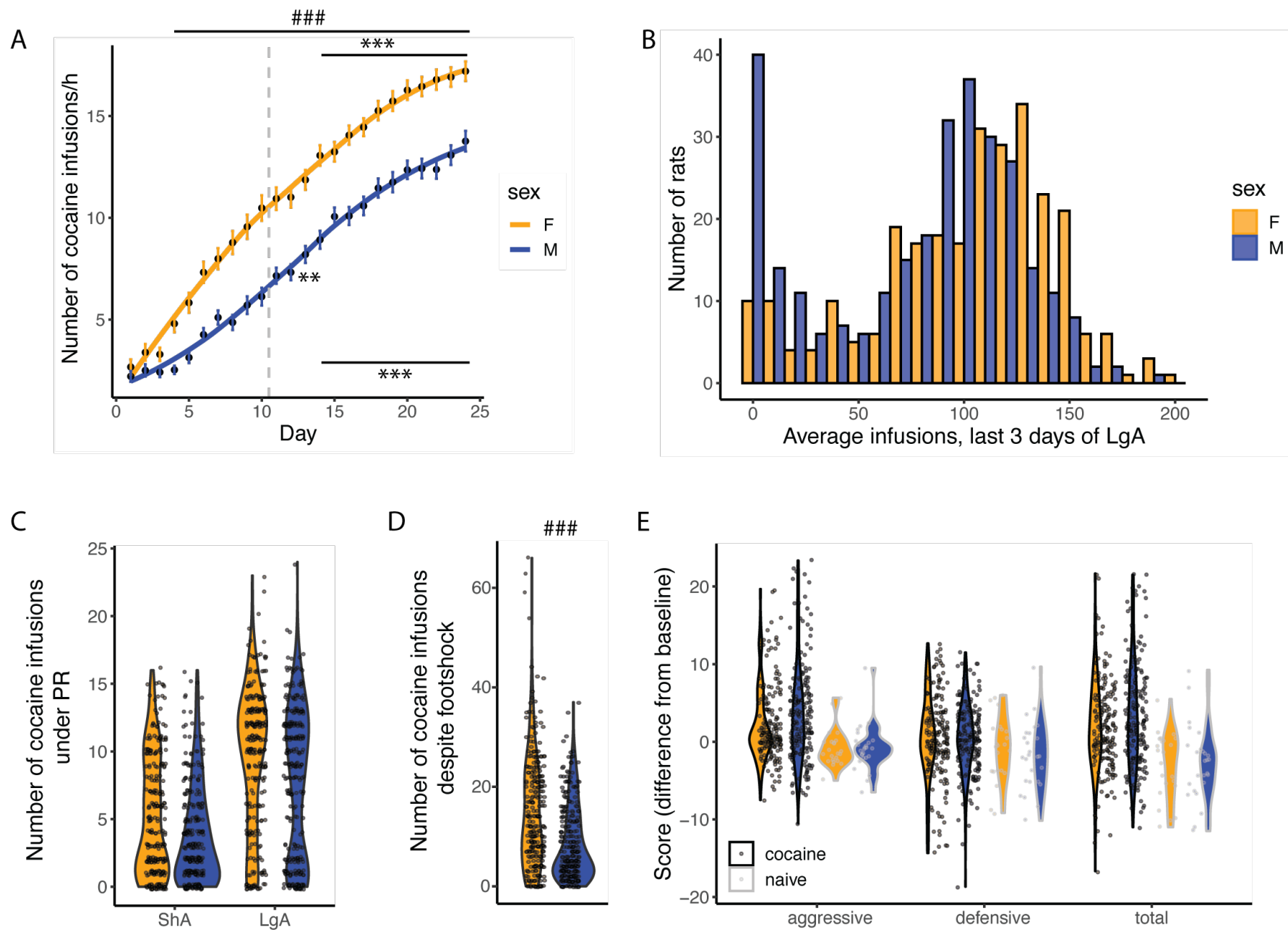

Figure 2. Sex differences in addiction-like behaviors. A) Cocaine infusions during short (2h, ShA) and long (6h, $\operatorname{LgA}$ ) access of cocaine self-administration ( $N=275 F$ and $292 M)$, ${ }^{* *} p<0.01$ vs day 1 of $L g A$ and \#\# $p<0.01$ vs males). B) Average number of daily infusions for the last 3 days of $L g A$ in male and female rats ( $N=275 F$ and $292 M)$. C) Violin plot of number of cocaine infusions under progressive ratio (PR) test after ShA and LgA (N=273 F and 291 M). D) Number of infusions despite footshock after $\operatorname{Lg} A\left(N=275 F\right.$ and $\left.292 M,{ }^{* * *} p<0.001\right)$. E) Increase in irritabilitylike behavior in males and females after $\operatorname{Lg} A$ vs baseline compared to naive rats, no significant sex differences were detected.

\section{Individual differences in cocaine addiction like behavior: characterization of 2 populations} of rats

While all HS rats acquired cocaine self-administration during the ShA phase, we detected major individual differences in their escalation profile that resulted in a bimodal distribution of cocaine intake. We identified animals that were resilient ( $\sim 20 \%$ of the entire population, they showed an 
hourly intake of less than 8 infusions/h, similar to the intake during ShA, Fig 1B-C) or vulnerable (intake higher than 8 infusions/h) to cocaine addiction-like behaviors (Fig 3A-B). When looking at the escalation patterns in vulnerable and resilient rats we found that the vulnerable group showed an escalation of cocaine intake starting from day 3 of extended access ( $p<0.001$ vs day 1, after 2-way ANOVA followed by Bonferroni post hoc), while the rats in the resilient group did not show escalation of intake over the course of the behavioral paradigm, keeping the same level of cocaine infusions (Fig. 3A). Major differences between vulnerable and resilient rats were also observed in the motivation to obtain cocaine $(P R)(p<0.001$, after 2 way ANOVA followed by Bonferroni post hoc, Fig 3B) and in the compulsive like responding (foot shock, $\mathrm{p}<0.001$, after t-test, Fig 3C), but not in the irritability-like behavior. The difference in escalation correlated with differences in the other addiction-like behaviors $(\mathrm{R}=0.6$, $\mathrm{p}<0.001$, for PR and $\mathrm{R}=0.37, \mathrm{p}<0.001$ for compulsivity), but did not correlate with irritability like behavior $(\mathrm{R}=0.048, \mathrm{p}=\mathrm{NS})$. 

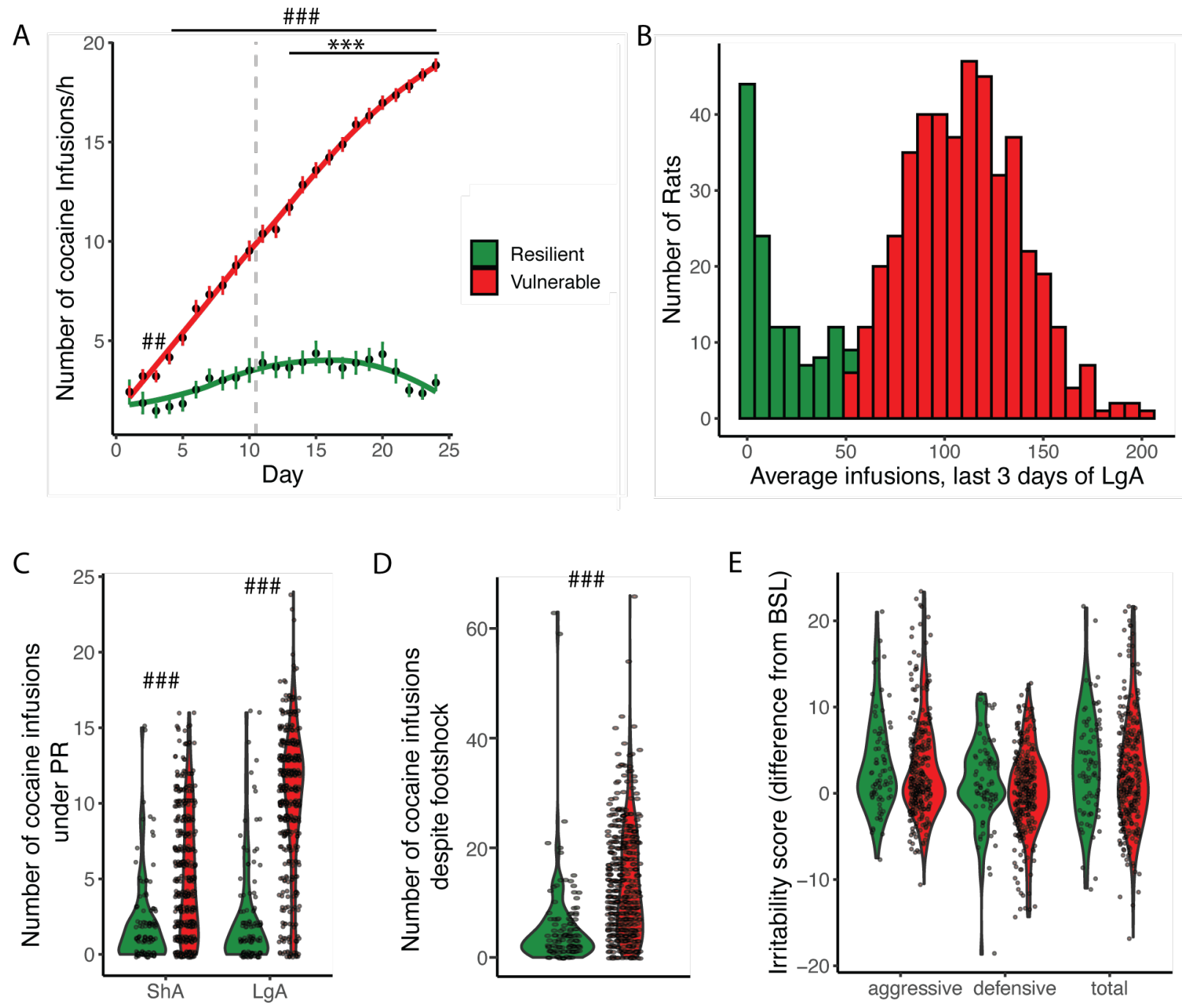

Figure 3. Correlation between the different addiction-like behaviors in resilient and vulnerable HS

rats. A) Number of cocaine infusions during short (2h, ShA) and long (6h, LgA) access of cocaine self-administration in resilient and vulnerable animals ( $N=567,{ }^{* * *} p<0.001$ vs $\operatorname{LgA}$ day $1, \# \# p<0.001$ vs resilient). B) Average number of infusions over the last 3 days for the individual animals ( $N=122$ resilient and 445 vulnerable). $C)$ Number of cocaine infusions under progressive ratio $(P R)$ at the end of ShA and $L g A$ ( $N=122$ resilient and 445 vulnerable, $\# \# p<0.001$ vs resilient). D) Number of infusions despite footshock after $\operatorname{LgA}(\mathrm{N}=122$ resilient and 445 vulnerable, \#\# $p<0.001$ vs resilient). E) Difference in irritability scores after $L g A$ and at baseline ( $N=79$ resilient, 301 vulnerable).

\section{Addiction Index: evaluation of individual differences in addiction-like behaviors.}

To take advantage of all the behaviors related to compulsive intake and withdrawal and further identify subjects that are consistently vulnerable $v s$. resilient to compulsive cocaine use, each measure was normalized into an index using its $Z$-score $\left(Z=\frac{x-\mu}{\sigma}\right)$, where $\chi$ is the raw value, $\mu$ is 
the mean of the cohort, and $\sigma$ is the standard deviation of the cohort. We thus obtained an FR Index, PR Index, and the irritability Index. Figure 4A-D shows the z-scores for escalation, motivation, compulsivity and irritability-like behavior. A principal component analysis incorporating the Z-scores of the 4 behavioral measures showed that almost half of the variability (48\%) can be explained by a single principal component (PC), which identified vulnerable and resilient rats and aligned with the escalation, motivation, and compulsivity Z-scores (eigenvalue $>1$, explaining $48 \%$ of variance and to which all three behaviors contributed in a valuable way, $r$ $=0.52$ to 0.60, Fig. $4 \mathrm{E}$ ). The irritability Z-score was almost exactly perpendicular to it, indicating that there was little to no correlation with the other addiction-like behavioral measures ( $r=$ 0.008). For this reason, we calculated an Addiction Index, that provides a comprehensive evaluation of compulsive cocaine use, by averaging the Z-scores of the three dependent variables that are explain almost 50\% of the variance, intake, PR and shock, leaving out irritability (Fig. 4F). Using the addiction index, animals can be ranked from low to high addiction-like behaviors and be categorized amongst animals with low (LA) or high (HA) addiction-like behaviors. 

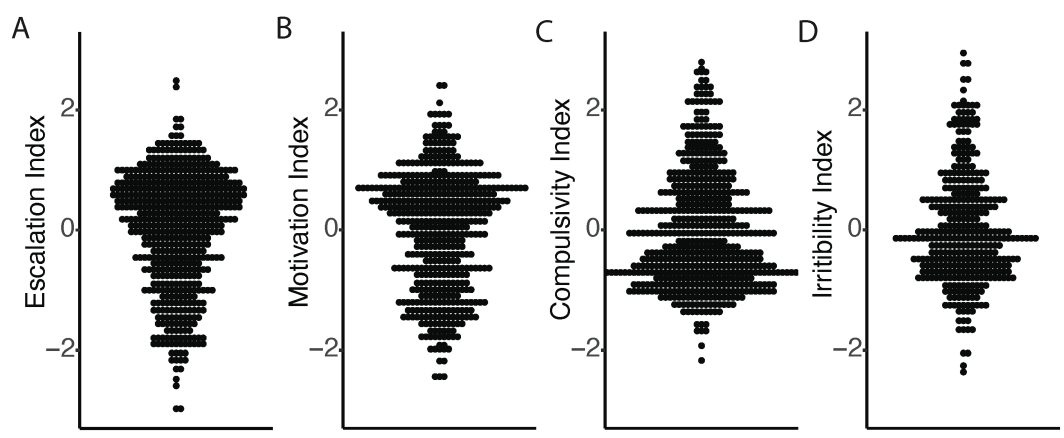

E

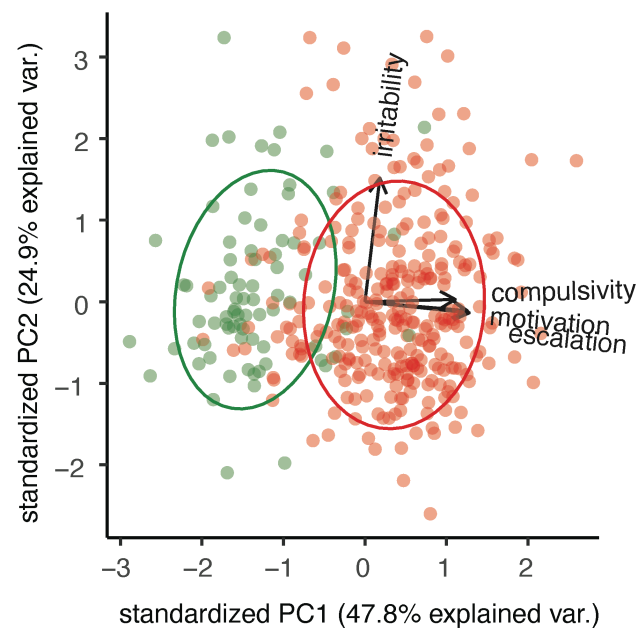

F

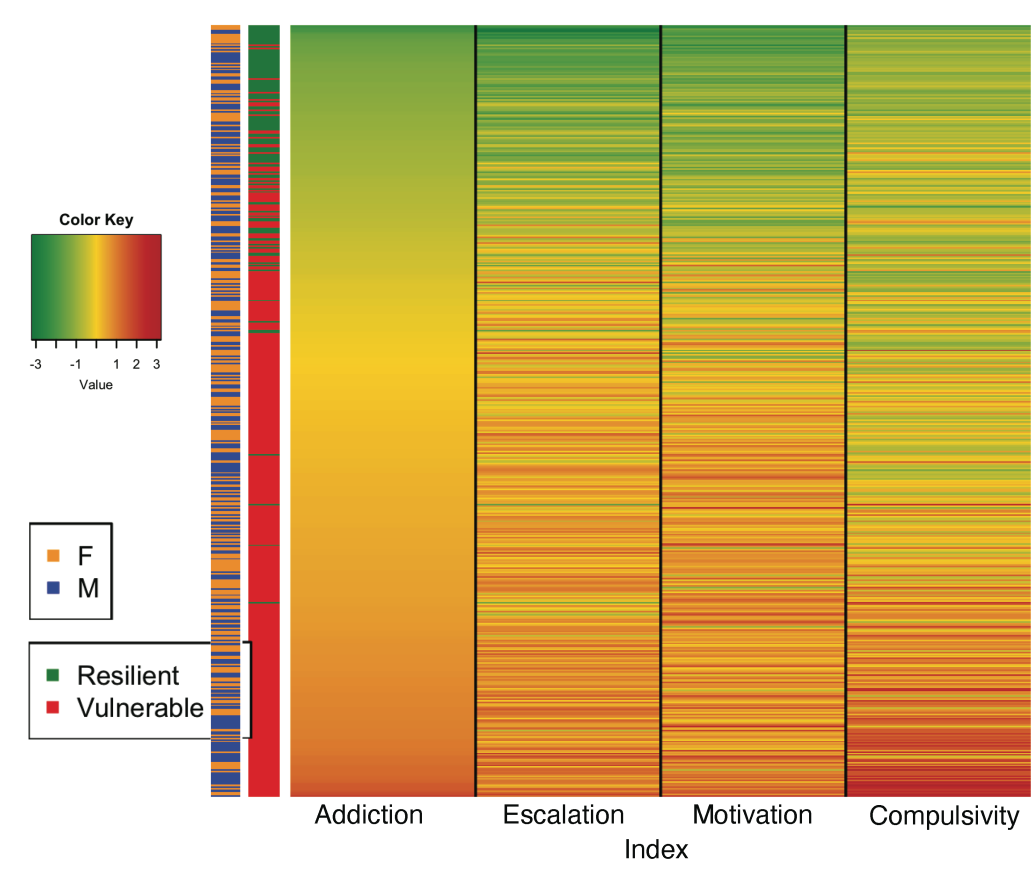


Figure 4. Normalizing and combining addiction like behaviors into an addiction index. A) Z-score for escalation, B) motivation, C) compulsivity and D) irritability in the whole population ( $N=566)$. E) Representation of the individual rats, resilient (green) or vulnerable (red) along the two first principal components, based on escalation, motivation, compulsivity and irritability z-scores. F) Representation of the addiction index for the individual rats with the constituting individual z-scores and their identification as resilient or vulnerable and male or female.

\section{Different degrees of addiction-like behavior}

The addiction index can be used to differentiate between mild, moderate and severe addiction like behavior in the vulnerable animals, by dividing them in 3 equal thirds. The different groups of rats, divided according to their intake over the last 3 days of $\mathrm{LgA}$ is shown in figure 5B. Cocaine intake was increased from resilient over mild and moderate to severe groups. The twoway ANOVA with group as between factor and sessions as within factor showed a significant effect of group ( $p<0.001)$, sessions $(p<0.001)$ and of the interaction group*sessions $(p<0.001)$. Pairwise comparisons for the significant main effects of groups, demonstrated that each of the subgroups (divided based on their Addiction Index) obtained significantly more cocaine of their immediate lower subgroup (severe $>$ moderate $>$ mild $>$ resilient, Fig 6A). In the progressive ratio test (Fig. 6C), the 2-way ANOVA showed a significant difference between the groups ( $p<$ 0.001). Pairwise comparisons for the significant main effect of groups showed significant differences between all the groups, with each of the subgroups showing increased motivation for cocaine compared to their immediate lower subgroup (severe $>$ moderate $>$ mild $>$ resilient, after One-way ANOVA followed by Bonferroni post hoc, Fig 6C). The difference between the groups shifts with compulsivity (Fig. 5D). For the compulsivity test the four groups show a similar pattern, with each of the subgroups showing increased compulsivity compared to their immediate subgroup $(\mathrm{p}<0.001$ after one-way ANOVA followed by the Bonferroni post hoc test). However, no significant difference was detected between the mild and resilient groups ( $p=$ $0.219)$. 
A

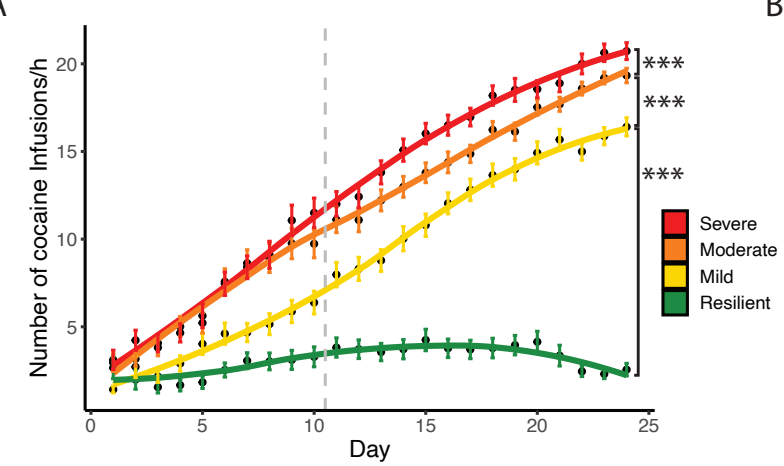

C

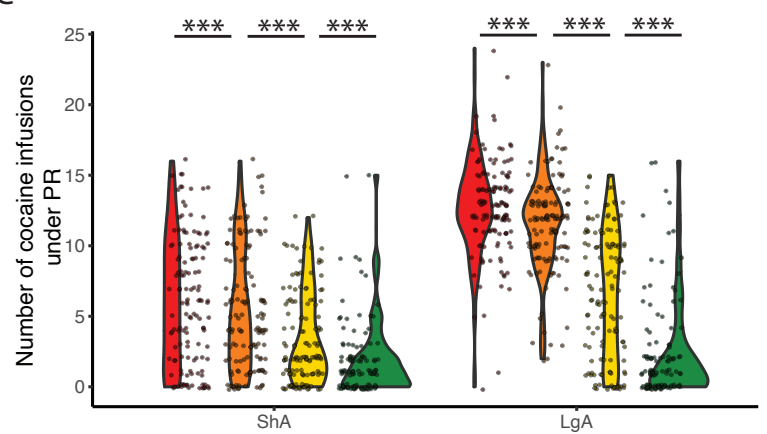

B

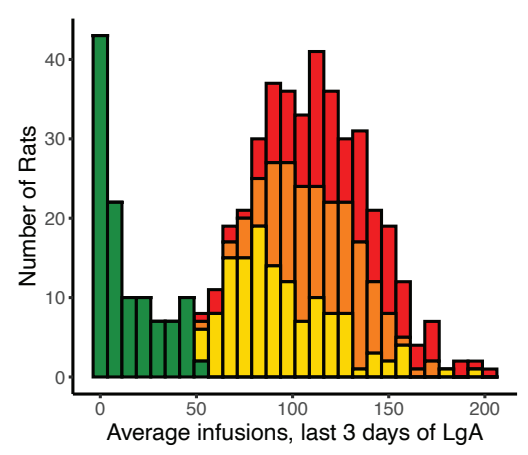

D

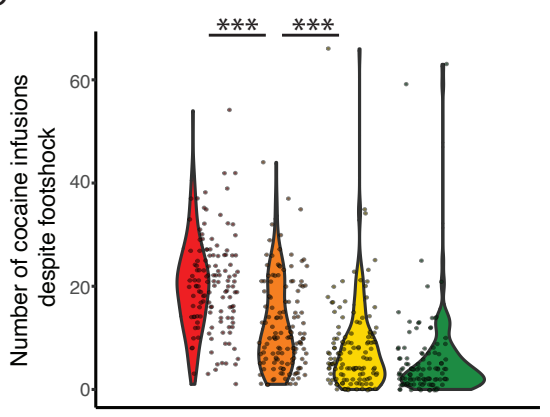

Figure 5. Different degrees of vulnerability to cocaine addictive behaviors. A) Cocaine infusions during short $(2 h, S h A)$ and long (6h, LgA) access of cocaine self-administration $\left({ }^{* * *} p<0.001\right)$. B) Average number of cocaine infusions over the last 3 days for the individual animals in the resilient, mild, moderate and severe vulnerable groups. C) Number of cocaine infusions under progressive ratio (PR) test at the end of the ShA and LgA phases (*** $p$ $<0.001)$. D) Number of infusions despite footshock after LgA for the resilient and vulnerable groups $\left({ }^{* * *} p<0.001\right)$.

\section{Discussion}

We characterized addiction like behavior in over 550 genetically diverse HS rats by establishing a large behavioral screening problem aimed at studying different cocaine related behaviors (escalation, motivation, compulsivity and irritability). We employed a high throughput behavioral paradigm using a state-of-the-art model of extended access to intravenous selfadministration combined with behavioral characterization of compulsivity using progressive ratio responding and responding despite adverse consequences (contingent foot shocks). We choose this model because it is highly relevant to cocaine use disorder (Edwards \& Koob, 2013; Olivier George, George F Koob, \& Leandro F Vendruscolo, 2014), and is associated with neuroadaptations that are also observed in humans with cocaine use disorder (Adinoff et al., 1990; Briand, Flagel, Seeman, \& Robinson, 2008; Olivier George, Chitra D. Mandyam, Sunmee 
Wee, \& George F. Koob, 2008; George et al., 2012; Vendruscolo et al., 2012). There is now compelling evidence that escalation of cocaine intake using this protocol is associated with compulsive cocaine use as measured by escalation of intake, increased progressive ratio responding and increased resistance to adverse consequence (foot shocks). The escalation model has been shown to exhibit 7 of the 11 items in the DSM-V, including most of the criteria required for severe use disorder: 1) Tolerance (Ben-Shahar, Moscarello, \& Ettenberg, 2006), 2) Withdrawal (Ahmed, Kenny, Koob, \& Markou, 2002), 3) Substance taken in larger amount than intended (Ahmed \& Koob, 1998), 4) Unsuccessful efforts to quit (Ahmed \& Cador, 2006; Lenoir, Serre, Cantin, \& Ahmed, 2007), 5) Considerable time spent to obtain the drug (Wee, Mandyam, Lekic, \& Koob, 2008), 6) Important social, work or recreational activities given up because of use (Olivier George et al., 2008; Lenoir, Cantin, Vanhille, Serre, \& Ahmed, 2013), 7) Continued use despite adverse consequence (Seif et al., 2013; Vanderschuren \& Everitt, 2004; Vendruscolo et al., 2012). Previous studies have employed smaller numbers of animals, used inbred strains that were selectively bred to be high or low responders (Z. Zhou et al., 2019) and used the BXD recombinant inbred mouse panel (Dickson et al., 2016) or Hybrid Mouse Diversity Panel (HMDP) (Bagley, Arshad, Smith, \& Jentsch, 2021). Moreover, several of these studies also used other proxies for addiction-like behaviors, like exploratory locomotion (Z. Zhou et al., 2019) or cocaine-induced motor effects (Vorspan et al., 2020) following passive cocaine injections. This is the first high-throughput intravenous cocaine self-administration study in HS rats, a unique genetically diverse population that self-administer cocaine with high interindividual variability and low intra-individual variability. Such behavioral variability betweensubjects and consistency within subjects will maximize the identification of gene variants associated with compulsive cocaine use.

The evaluation of different addiction-like behaviors is important as multiple elements of addiction vulnerability were found to be independently heritable (Eid, Pullmann, LI, Thomas, \& Jhou, 2019), and are likely controlled by distinct genes that remain to be identified. Inbred strains studies have clearly identified massive strain differences in the propensity for escalation of cocaine use, which speaks to its heritability. Davis et al. (Davis, Clinton, Akil, \& Becker, 2008) tested selectively bred rats $(\mathrm{HR} / \mathrm{LR})$ and found that HR rats acquired cocaine self-administration more rapidly than LR rats. Picetti et al (Picetti, Ho, Butelman, \& Kreek, 2010) showed that Lewis rats strongly preferred higher doses of cocaine in this paradigm, whereas Fischer preferred 
much smaller doses; in a follow-up study Valenza et al (Valenza, Picetti, Yuferov, Butelman, \& Kreek, 2016) found Lewis and Fischer were starkly different for escalation of cocaine selfadministration. The differences between these two strains were recently reviewed by Caroni (Cadoni \& Di Chiara, 2007). Finally, Meyer et al. assessed 12 inbred rat strains for various traits including intravenous amphetamine self-administration (acquisition, extinction, and amphetamine-induced reinstatement) and found widespread and highly significant strain differences (Meyer et al., 2010). Thus, there is ample evidence of heritable differences in compulsive cocaine use in various inbred rat strains. In our work, we found that escalation, motivation, and compulsivity were typically very similar within subject, while there was a large variability between subjects (Fig .4F). Half of this variability could be captured in one dimension through a composite measure of escalation, motivation, and compulsivity. To minimize cohortspecific effects, large cohorts $(n=46-60)$ were used and the behavior normalized within cohorts using a $Z$-score. Intake after escalation was bimodally distributed. While $80 \%$ of animals escalated their intake as expected, $20 \%$ of resilient rats maintained a low intake. This was not due to lack of catheter patency or to a failure to acquire self-administration and it seem specific to cocaine, since we recently demonstrated that HS rats acquire simple food self-administration very well (Sedighim et al., 2021).

By computing an Addiction Index, we were able to better characterize the addiction-like behaviors in the vulnerable population by distinguishing their addiction phenotype in mild, moderate, and severe. Mild vulnerable animals showed low escalation of intake, but had similar motivation for cocaine and compulsivity to the resilient animals. Severe vulnerable animals showed very strong escalation of intake, high motivation, and compulsivity. The moderate vulnerable animals were intermediate in all measures. Withdrawal-induced irritability-like behavior did not correlate with any of these behaviors. As correlation of withdrawal-induced irritability-like behavior with intake is expected, this probably indicates that the bottlebrush test did not properly capture cocaine-induced withdrawal.

Previous studies have also shown significant sex differences in addiction. While cocaine use disorder is more prevalent in males than in females (SAMHSA, 2019), women may be more vulnerable to the reinforcing effects of cocaine, acquire cocaine abuse faster and at higher levels than males (Cummings et al., 2011; Jackson, Robinson, \& Becker, 2006). Sex differences were 
found in HS rats for cocaine cue preference (King et al., 2021). With our large dataset we were able to replicate and confirm these known effects, further contributing to the importance in studying the origin of these differences in addiction-like behavior. We found that female rats self-administered more cocaine and showed greater motivation and compulsive-like behavior. The increased motivation and compulsivity in females cannot simply be explained by the higher intake amongst females, as the significant difference remains when we compared only the vulnerable rats in both sexes (see Fig. S1).

The behavioral characterization of HS rats was the first step in characterizing and understanding individual differences in a genetically diverse population. Future studies will combine these differences with the animals' genetic and genomic data obtained through whole genome sequencing to perform a GWAS aimed at investigate gene variants that contribute to vulnerability and resilience to cocaine addiction-like behavior (Chitre et al., 2020). This will then allow for the identification of specific genetic loci, estimatation of heritability, investigation of genetic correlations and performance of phenome-wide association studies (PheWAS) and transcriptome-wide association studies (TWAS). All these studies will inform on individual differences in vulnerability and resilience to the addiction phenotype and will provide different addiction-like traits for human personalized diagnosis and novel treatment approaches. Finally, to facilitate investigating the biological origins of the difference in cocaine addiction-like behavior through epigenetic, transcriptomic (Vorspan et al., 2020), microbiomic, neurobiological or other mechanisms between resilient and vulnerable groups or males and females beyond genetic factors, samples of these behavioral and genetically characterized animals are collected and available free of charge through the cocaine biobank (Carrette et al., 2021).

\section{Acknowledgements}

This work was supported by National Institutes of Health grant DA043799 from the National Institute on Drug Abuse. The authors declare no competing financial interests.

\section{References}


Adinoff, B., Martin, P. R., Bone, G. H., Eckardt, M. J., Roehrich, L., George, D. T., . . Gold, P. W. (1990). Hypothalamic-pituitary-adrenal axis functioning and cerebrospinal fluid corticotropin releasing hormone and corticotropin levels in alcoholics after recent and long-term abstinence. Arch Gen Psychiatry, 47(4), 325-330.

Agrawal, A., Verweij, K. J., Gillespie, N. A., Heath, A. C., Lessov-Schlaggar, C. N., Martin, N. G., . . Lynskey, M. T. (2012). The genetics of addiction-a translational perspective. Transl Psychiatry, 2, e140. doi: 10.1038/tp.2012.54

Ahmed, S. H., \& Cador, M. (2006). Dissociation of psychomotor sensitization from compulsive cocaine consumption. Neuropsychopharmacology, 31(3), 563-571. doi: 10.1038/sj.npp.1300834

Ahmed, S. H., Kenny, P. J., Koob, G. F., \& Markou, A. (2002). Neurobiological evidence for hedonic allostasis associated with escalating cocaine use. Nat Neurosci, 5(7), 625-626. doi: $10.1038 / \mathrm{nn} 872$

Ahmed, S. H., \& Koob, G. F. (1998). Transition from moderate to excessive drug intake: change in hedonic set point. Science, 282(5387), 298-300.

Bagley, J. R., Arshad, H. K., Smith, D. J., \& Jentsch, J. D. (2021). Extreme Phenotypic Diversity in Operant Responding for an Intravenous Cocaine or Saline Infusion in the Hybrid Mouse Diversity Panel. Biorxiv. doi: https://doi.org/10.1101/2021.02.03.429584

Ben-Shahar, O., Moscarello, J. M., \& Ettenberg, A. (2006). One hour, but not six hours, of daily access to self-administered cocaine results in elevated levels of the dopamine transporter. Brain Res, 1095(1), 148-153. doi: 10.1016/j.brainres.2006.04.002

Briand, L. A., Flagel, S. B., Seeman, P., \& Robinson, T. E. (2008). Cocaine self-administration produces a persistent increase in dopamine D2 High receptors. Eur Neuropsychopharmacol, 18(8), 551-556. doi: 10.1016/j.euroneuro.2008.01.002

Briand, L. A., Gross, J. P., \& Robinson, T. E. (2008). Impaired object recognition following prolonged withdrawal from extended-access cocaine self-administration. Neuroscience, 155(1), 1-6. doi: 10.1016/j.neuroscience.2008.06.004

Butler, A. J., Rehm, J., \& Fischer, B. (2017). Health outcomes associated with crack-cocaine use: Systematic review and meta-analyses. Drug Alcohol Depend, 180, 401-416. doi: 10.1016/j.drugalcdep.2017.08.036

Cadoni, C., \& Di Chiara, G. (2007). Differences in dopamine responsiveness to drugs of abuse in the nucleus accumbens shell and core of Lewis and Fischer 344 rats. J Neurochem, 103(2), 487-499. doi: 10.1111/j.1471-4159.2007.04795.x

Carrette, L. L., de Guglielmo, G., Kallupi, M., Maturin, L., Brennan, M., Boomhower, B., . . . George, O. (2021). The cocaine and oxycodone biobanks, two repositories from genetically diverse and behaviorally characterized rats for the study of addiction. eNeuro. doi: 10.1523/ENEURO.0033-21.2021

Chitre, A. S., Polesskaya, O., Holl, K., Gao, J., Cheng, R., Bimschleger, H., . . . Solberg Woods, L. C. (2020). Genome-Wide Association Study in 3,173 Outbred Rats Identifies Multiple Loci for Body Weight, Adiposity, and Fasting Glucose. Obesity (Silver Spring), 28(10), 1964-1973. doi: 10.1002/oby.22927

Cummings, J. A., Gowl, B. A., Westenbroek, C., Clinton, S. M., Akil, H., \& Becker, J. B. (2011). Effects of a selectively bred novelty-seeking phenotype on the motivation to take cocaine in male and female rats. Biol Sex Differ, 2, 3. doi: 10.1186/2042-6410-2-3 
Davis, B. A., Clinton, S. M., Akil, H., \& Becker, J. B. (2008). The effects of novelty-seeking phenotypes and sex differences on acquisition of cocaine self-administration in selectively bred High-Responder and Low-Responder rats. Pharmacol Biochem Behav, 90(3), 331-338. doi: 10.1016/j.pbb.2008.03.008

Dickson, P. E., Miller, M. M., Calton, M. A., Bubier, J. A., Cook, M. N., Goldowitz, D., . . . Mittleman, G. (2016). Systems genetics of intravenous cocaine self-administration in the BXD recombinant inbred mouse panel. Psychopharmacology (Berl), 233(4), 701-714. doi: $10.1007 / \mathrm{s} 00213-015-4147-\mathrm{z}$

Ducci, F., \& Goldman, D. (2012). The genetic basis of addictive disorders. Psychiatr Clin North Am, 35(2), 495-519. doi: 10.1016/j.psc.2012.03.010

Edwards, S., \& Koob, G. F. (2013). Escalation of drug self-administration as a hallmark of persistent addiction liability. Behav Pharmacol, 24(5-6), 356-362. doi: 10.1097/FBP.0b013e3283644d15

Eid, M., Pullmann, D., LI, H., Thomas, A., \& Jhou, T. C. (2019). Multiple elements of addiction vulnerability are independently heritable in rats. Biorxiv. doi:

https://doi.org/10.1101/566018

Erzurumluoglu, A. M., Liu, M., Jackson, V. E., Barnes, D. R., Datta, G., Melbourne, C. A., . . . Howson, J. M. M. (2020). Meta-analysis of up to 622,409 individuals identifies 40 novel smoking behaviour associated genetic loci. Mol Psychiatry, 25(10), 2392-2409. doi: 10.1038/s41380-018-0313-0

George, O., Koob, G. F., \& Vendruscolo, L. F. (2014). Negative reinforcement via motivational withdrawal is the driving force behind the transition to addiction. Psychopharmacology (Berl), 231(19), 3911-3917. doi: 10.1007/s00213-014-3623-1

George, O., Koob, G. F., \& Vendruscolo, L. F. (2014). Negative reinforcement via motivational withdrawal is the driving force behind the transition to addiction. Psychopharmacology, 231(19), 3911-3917. doi: 10.1007/s00213-014-3623-1

George, O., Mandyam, C. D., Wee, S., \& Koob, G. F. (2008). Extended access to cocaine selfadministration produces long-lasting prefrontal cortex-dependent working memory impairments. Neuropsychopharmacology, 33(10), 2474-2482. doi: 10.1038/sj.npp.1301626

George, O., Mandyam, C. D., Wee, S., \& Koob, G. F. (2008). Extended access to cocaine selfadministration produces long-lasting prefrontal cortex-dependent working memory impairments. Neuropsychopharmacology, 33(10), 2474-2482. doi: 10.1038/sj.npp.1301626

George, O., Sanders, C., Freiling, J., Grigoryan, E., Vu, S., Allen, C. D., . . Koob, G. F. (2012). Recruitment of medial prefrontal cortex neurons during alcohol withdrawal predicts cognitive impairment and excessive alcohol drinking. Proceedings of the National Academy of Sciences of the United States of America, 109(44), 18156-18161. doi: 10.1073/pnas.1116523109

Hart, A. B., de Wit, H., \& Palmer, A. A. (2013). Candidate gene studies of a promising intermediate phenotype: failure to replicate. Neuropsychopharmacology, 38(5), 802-816. doi: 10.1038/npp.2012.245

Hedegaard, H., Bastian, B. A., Trinidad, J. P., Spencer, M., \& Warner, M. (2018). Drugs Most Frequently Involved in Drug Overdose Deaths: United States, 2011-2016. Natl Vital Stat Rep, 67(9), 1-14. 
Jackson, L. R., Robinson, T. E., \& Becker, J. B. (2006). Sex differences and hormonal influences on acquisition of cocaine self-administration in rats. Neuropsychopharmacology, 31(1), 129-138. doi: 10.1038/sj.npp.1300778

Kallupi, M., Carrette, L. L. G., Kononoff, J., Solberg Woods, L. C., Palmer, A. A., Schweitzer, P., . . . de Guglielmo, G. (2020). Nociceptin attenuates the escalation of oxycodone selfadministration by normalizing CeA-GABA transmission in highly addicted rats. Proc Natl Acad Sci U S A, 117(4), 2140-2148. doi: 10.1073/pnas.1915143117

Kampman, K. M. (2019). The treatment of cocaine use disorder. Sci Adv, 5(10), eaax1532. doi: 10.1126/sciadv.aax 1532

Kimbrough, A., de Guglielmo, G., Kononoff, J., Kallupi, M., Zorrilla, E. P., \& George, O. (2017a). CRF1 Receptor-Dependent Increases in Irritability-Like Behavior During Abstinence from Chronic Intermittent Ethanol Vapor Exposure. Alcohol Clin Exp Res, 41(11), 1886-1895. doi: 10.1111/acer.13484

Kimbrough, A., de Guglielmo, G., Kononoff, J., Kallupi, M., Zorrilla, E. P., \& George, O. (2017b). CRF(1) Receptor-Dependent Increases in Irritability-Like Behavior During Abstinence from Chronic Intermittent Ethanol Vapor Exposure. Alcohol Clin Exp Res, 41(11), 1886-1895. doi: 10.1111/acer.13484

King, C. P., Tripi, J. A., Hughson, A. R., Horvath, A. P., Lamparelli, A. C., Holl, K. L., . . . Meyer, P. J. (2021). Sensitivity to food and cocaine cues are independent traits in a large sample of heterogeneous stock rats. Sci Rep, 11(1), 2223. doi: 10.1038/s41598-02080798-w

Klepstad, P., Fladvad, T., Skorpen, F., Bjordal, K., Caraceni, A., Dale, O., . . . the European Association for Palliative Care Research, N. (2011). Influence from genetic variability on opioid use for cancer pain: a European genetic association study of 2294 cancer pain patients. Pain, 152(5), 1139-1145. doi: 10.1016/j.pain.2011.01.040

Kreek, M. J., Bart, G., Lilly, C., LaForge, K. S., \& Nielsen, D. A. (2005). Pharmacogenetics and human molecular genetics of opiate and cocaine addictions and their treatments. Pharmacol Rev, 57(1), 1-26. doi: 10.1124/pr.57.1.1

Lenoir, M., Cantin, L., Vanhille, N., Serre, F., \& Ahmed, S. H. (2013). Extended heroin access increases heroin choices over a potent nondrug alternative. Neuropsychopharmacology, 38(7), 1209-1220. doi: 10.1038/npp.2013.17

Lenoir, M., Serre, F., Cantin, L., \& Ahmed, S. H. (2007). Intense sweetness surpasses cocaine reward. PLoS One, 2(8), e698. doi: 10.1371/journal.pone.0000698

Meyer, A. C., Rahman, S., Charnigo, R. J., Dwoskin, L. P., Crabbe, J. C., \& Bardo, M. T. (2010). Genetics of novelty seeking, amphetamine self-administration and reinstatement using inbred rats. Genes Brain Behav, 9(7), 790-798. doi: 10.1111/j.1601183X.2010.00616.X

Picetti, R., Ho, A., Butelman, E. R., \& Kreek, M. J. (2010). Dose preference and dose escalation in extended-access cocaine self-administration in Fischer and Lewis rats. Psychopharmacology (Berl), 211(3), 313-323. doi: 10.1007/s00213-010-1899-3

SAMHSA, A. (2019). Results from the 2018 National Survey on Drug Use and Health: Summary of national findings. Annual Report.

Sanchez-Roige, S., Fontanillas, P., Elson, S. L., andMe Research, T., Gray, J. C., de Wit, H., .. . Palmer, A. A. (2019). Genome-wide association study of alcohol use disorder identification test (AUDIT) scores in 20328 research participants of European ancestry. Addict Biol, 24(1), 121-131. doi: 10.1111/adb.12574 
Sanchez-Roige, S., Palmer, A. A., Fontanillas, P., Elson, S. L., andMe Research Team, t. S. U. D. W. G. o. t. P. G. C., Adams, M. J., . . . Clarke, T. K. (2019). Genome-Wide Association Study Meta-Analysis of the Alcohol Use Disorders Identification Test (AUDIT) in Two Population-Based Cohorts. Am J Psychiatry, 176(2), 107-118. doi: 10.1176/appi.ajp.2018.18040369

Sedighim, S., Carrette, L. L., Venniro, M., Shaham, Y., de Guglielmo, G., \& George, O. (2021). Individual difference in addiction-like behaviors and choice between cocaine versus food in Heterogeneous Stock rats. Biorxiv.

Seif, T., Chang, S. J., Simms, J. A., Gibb, S. L., Dadgar, J., Chen, B. T., . . Hopf, F. W. (2013). Cortical activation of accumbens hyperpolarization-active NMDARs mediates aversionresilient alcohol intake. Nat Neurosci, 16(8), 1094-1100. doi: 10.1038/nn.3445

Solberg Woods, L. C., \& Palmer, A. A. (2019). Using Heterogeneous Stocks for Fine-Mapping Genetically Complex Traits. Methods Mol Biol, 2018, 233-247. doi: 10.1007/978-1-49399581-3_11

Valenza, M., Picetti, R., Yuferov, V., Butelman, E. R., \& Kreek, M. J. (2016). Strain and cocaine-induced differential opioid gene expression may predispose Lewis but not Fischer rats to escalate cocaine self-administration. Neuropharmacology, 105, 639-650. doi: 10.1016/j.neuropharm.2016.01.004

Vanderschuren, L. J., \& Everitt, B. J. (2004). Drug seeking becomes compulsive after prolonged cocaine self-administration. Science, 305(5686), 1017-1019. doi: 10.1126/science.1098975

Vendruscolo, L. F., Barbier, E., Schlosburg, J. E., Misra, K. K., Whitfield, T. W., Jr., Logrip, M. L., . . Koob, G. F. (2012). Corticosteroid-dependent plasticity mediates compulsive alcohol drinking in rats. $J$ Neurosci, 32(22), 7563-7571. doi: 10.1523/JNEUROSCI.006912.2012

Vorspan, F., Icick, R., Mekdad, N., Courtin, C., Bloch, V., Bellivier, F., . . Benturquia, N. (2020). Translational study of the whole transcriptome in rats and genetic polymorphisms in humans identifies LRP1B and VPS13A as key genes involved in tolerance to cocaineinduced motor disturbances. Transl Psychiatry, 10(1), 381. doi: 10.1038/s41398-02001050-7

Vowles, K. E., McEntee, M. L., Julnes, P. S., Frohe, T., Ney, J. P., \& van der Goes, D. N. (2015). Rates of opioid misuse, abuse, and addiction in chronic pain: a systematic review and data synthesis. Pain, 156(4), 569-576. doi: 10.1097/01.j.pain.0000460357.01998.f1

Walters, R. K., Polimanti, R., Johnson, E. C., McClintick, J. N., Adams, M. J., Adkins, A. E., . . . Agrawal, A. (2018). Transancestral GWAS of alcohol dependence reveals common genetic underpinnings with psychiatric disorders. Nat Neurosci, 21(12), 1656-1669. doi: 10.1038/s41593-018-0275-1

Wee, S., Mandyam, C. D., Lekic, D. M., \& Koob, G. F. (2008). Alpha 1-noradrenergic system role in increased motivation for cocaine intake in rats with prolonged access. Eur Neuropsychopharmacol, 18(4), 303-311. doi: 10.1016/j.euroneuro.2007.08.003

Zhou, H., Sealock, J. M., Sanchez-Roige, S., Clarke, T. K., Levey, D. F., Cheng, Z., . . . Gelernter, J. (2020). Genome-wide meta-analysis of problematic alcohol use in 435,563 individuals yields insights into biology and relationships with other traits. Nat Neurosci, 23(7), 809-818. doi: 10.1038/s41593-020-0643-5

Zhou, Z., Blandino, P., Yuan, Q., Shen, P. H., Hodgkinson, C. A., Virkkunen, M., .. . Goldman, D. (2019). Exploratory locomotion, a predictor of addiction vulnerability, is oligogenic in 
rats selected for this phenotype. Proc Natl Acad Sci U S A, 116(26), 13107-13115. doi: $10.1073 /$ pnas. 1820410116

\section{Supplementary Figure}

A

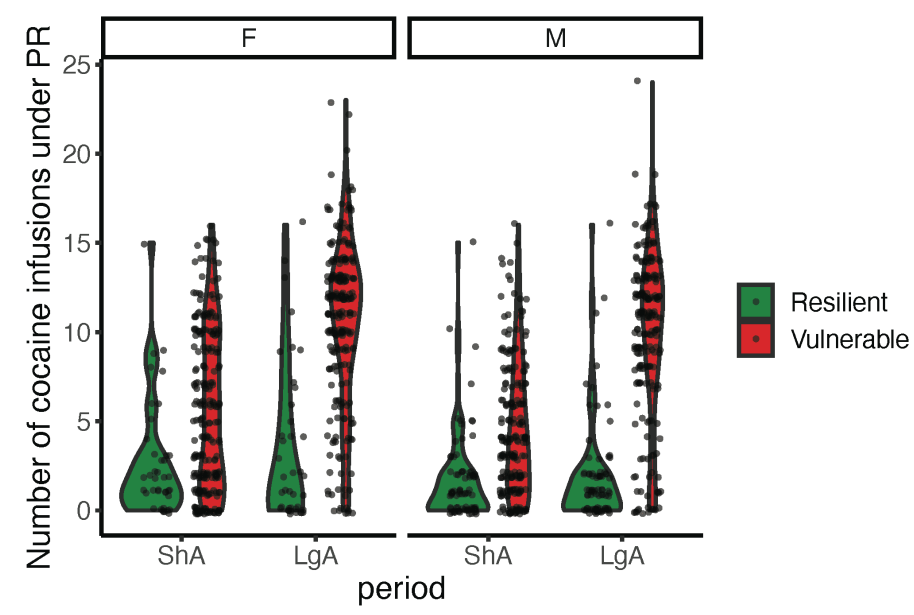

B

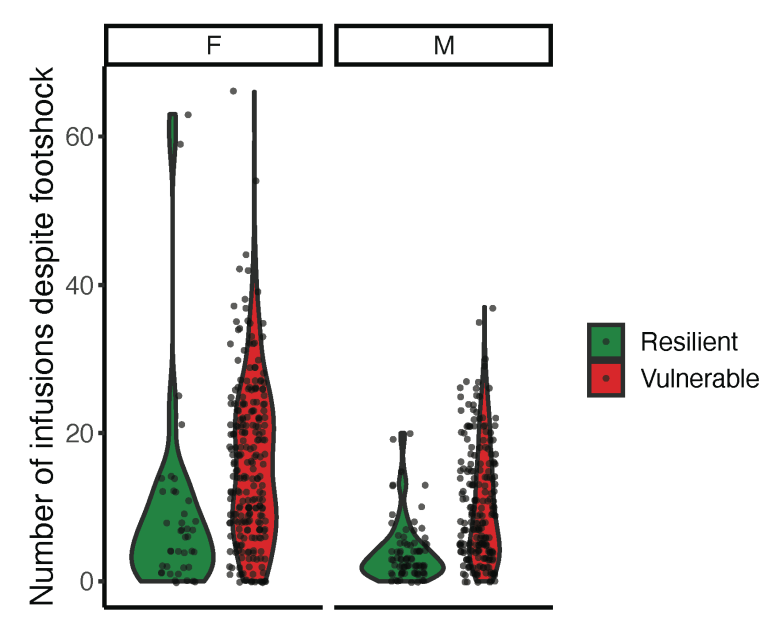

Figure S1. Motivation (A) and compulsivity (B) for the vulnerable and resilient populations split by sex 